九州大学学術情報リポジトリ

Kyushu University Institutional Repository

\title{
On the Determination of Sample Size from the Two Sample Theoritical Formulation
}

Kitagawa, Toshio

Kyushu University

Kitahara, Teisuke

Nomachi, Yukio

Kyushu University

Watanabe, Nobuo

https://doi.org/10.5109/12960

出版情報 : 統計数理研究. 5 (3/4)，pp. 35-45，1953-06. Research Association of Statistical Sciences

バージョン：

権利関係 : 


\title{
ON THE DETERMINATION OF SAMPLE SIZE FROM THE TWO SAMPLE THEORTICAL FORMULATION
}

\author{
$\mathrm{By}$ \\ Tosio Kitagawa, Teisuke Kitahara, \\ Yukio NomachI and Nobuo Watanabe
}

\section{\$1. Two sample theoretical formulation.}

Let us consider a normal population $N\left(\boldsymbol{m}, \sigma^{2}\right)$ with unknown mean $\boldsymbol{m}$ and unknown variance $\sigma^{2}$, and let $O_{n}:\left(x_{1}, x_{2}, \cdots, x_{n}\right)$ be a random sample of size $n$ from this population. Then, as well known, the confidence interval for the mean $m$ with confidence coefficient $1-\alpha$ is given by

$$
\left(\bar{x}-t_{n-1}(\alpha) s n^{-1 / 2}, \quad x+t_{n-1}(\alpha) s n^{-1 / 2}\right),
$$

where $\bar{x}$ and $s^{2}$ are the sample mean and unbiassed variance estimate from obtained from the sample respectively, and $t_{n-1}(\alpha)$ is the $\alpha$ significant level of $t$-distribution with the $n-1$ degrees of freedom. The length of confidence interval obtained in this way is $2 t_{n-1}(\alpha) s n^{-1 / 2}$, and the expectaion of this length is equal to $2 t_{n-1}(\alpha) E\{s\} n^{-1 / 2}=2 t_{n-1}(\alpha) \sigma n^{-1 / 2}+O\left(n^{-3 / 2}\right)$, which tends to zero as $n$ increases infinitely and moreover we have $4 t_{n-1}^{2}(\alpha)$ $n^{-1} \sigma^{2}\{s\}=2 t_{n-1}^{2}(\alpha) o^{2} n^{-2}+O\left(n^{-3}\right)$. In view of these properties of length of the condidence interval, it will be suggested that there may be some possibilities that a length of confidence interval with any assigned confidence coefficient $1-\alpha$ can be smaller than any assigned length $2 d$, by choosing the sample size sufficiently large. The real situations, however, should be more carefully formulated. There are two essential points which should be remarked. The first point is that the relation $t_{n-1}(\alpha) s n^{-1 / 2} \leq d$ cannot be established with the probability one, for any fixed $n$, while the second one is that since the population variance $\sigma^{2}$ is unknown to us, the equation such as $t_{n-1}(\alpha) s n^{-1 / 2} \leq \boldsymbol{d}$ cannot give us any definite procedure for determining the sampl esize $n$. In view of these two facts, let us now appeal to the two sample theoretical approach, which will suggest following type of two sample procedure: (i) Let us at first draw a sample of size $n_{1} O_{n 1}:\left(x_{1,1}, x_{1,2}, \ldots\right.$, $\left.x_{1}, n_{1}\right)$ from our population, and let us make the undiassed estimate $s_{1}^{2}$ for $\sigma^{2}$ :

$$
s_{1}^{2}=\left(n_{1}-1\right)^{-1} \sum_{j=1}^{n_{1}}\left(x_{1 j}-\bar{x}_{1}\right)^{2} .
$$

(ii) In view of this $s_{1}$, let us determine a size of second sample by some procedure, and draw a second sample of this size $O_{n_{2}}:\left(x_{21}, x_{22}, \cdots, x_{2 n_{2}}\right)$ from our population. Then our situation is to expect to satisfy the following relation: 
(1.03)

$$
\text { Pr. }\left\{t_{n_{2}-1}(\alpha) s_{2} n_{2}^{-1 / 2} \leq d\right\} \geq 1-\beta,
$$

where

$$
s_{2}{ }^{2}=\left(n_{2}-1\right)^{-1} \sum_{j=1}^{n_{2}}\left(x_{2 j}-\bar{x}_{2}\right)^{2} .
$$

It is to be noted that the sample size $n_{2}$ is itself a random variable as a function of the stochastic variables. In what follows let us denote the stochastic variable which represents the sample size $n_{2}$ by a bold type $\boldsymbol{n}_{2}$ in order to distinguish from a constant number $n_{2}$. In order that we may realize this type of two sample procedure, what remines to us to determine is how to select the size of the second sample $n_{2}$. Here let us appeal to the $F$-distribution which gives us, for two independent unbiassed variance estimates $s_{1}^{2}$ and $s_{2}^{2}$. with the degress of freedom $n_{1}-1$ and $n_{2}-1$ respectively.

$$
\text { Pr. }\left\{s_{2} / s_{1}<\left(F_{n_{1}-1}^{n_{2}-1}(\beta)\right)^{1 / 2}\right\}=1-\beta .
$$

In combination of (1.03) and (1.05) we have the relation

$$
\text { Pr. }\left\{s_{2} \leq \frac{d n_{2}^{1 / 2}}{t_{n_{2}-1}(\alpha)}\right\} \geqq 1-\beta=\operatorname{Pr} .\left\{s_{2} \leq s_{1} \sqrt{F_{n_{1}-1}^{n_{2}-1}(\beta)}\right\} .
$$

Therefore we propose to determine the size of second sample $n_{2}$ such as the following relations shall hold simultaneously:

$$
\frac{d n_{2}^{1 / 2}}{t_{n_{2}-1}(\alpha)} \geqq s_{1} \sqrt{F_{n_{1}^{-1}}^{n_{2}-1}(\beta)},
$$

and

$$
\frac{d\left(n_{2}-1\right)^{1 / 2}}{t_{n_{2}-2}(\alpha)}<s_{l} \sqrt{\bar{F}_{n_{1}-1}^{n_{2}-2}(\beta)}
$$

that is,

$$
\frac{d\left(n_{2}-1\right)^{1 / 2}}{t_{n_{2}-2}(\alpha) \sqrt{\bar{F}_{n_{1}^{2}-1}^{n_{2}-2}(\beta)}}<s_{1} \leq \frac{d n_{2}^{1 / 2}}{t_{n_{2}-1}(\alpha) \sqrt{F_{n_{1}-1}^{n_{2}-1}(\beta)}} .
$$

Consequently we have following relations :

$$
\begin{aligned}
& \text { Pr. }\left\{\frac{t_{n_{2}-1}(\alpha)}{\sqrt{n_{2}}} s_{2}<d\right\} \\
& \left.\left.=\sum_{n_{2}=3}^{\infty} \operatorname{Pr} .\right\} \frac{t_{n_{2}-1}(\alpha)}{\sqrt{n_{2}}} s_{2}<d / n_{2}\right\} \operatorname{Pr} .\left\{n_{2}=n_{2}\right\} \\
& =\sum_{n_{2}=3}^{\infty} \operatorname{Pr} \cdot\left\{\frac{d\left(n_{2}-1\right)^{1 / 2}}{t_{n_{2}-2}(\alpha) \sqrt{\boldsymbol{F}_{n_{1}-1}^{n_{2}-2}(\beta)}}<s_{1} \leq \frac{d n_{2}^{1 / 2}}{t_{n_{2}-1}(\alpha) \sqrt{\boldsymbol{F}_{n_{1}^{2}-1}^{n_{2}-1}(\beta)}}\right\} \\
& \text { - Pr. }\left\{\frac{t_{n_{2-1}(\alpha)}(\alpha)}{\sqrt{n}} s_{2}<d / s_{1}\right\}
\end{aligned}
$$




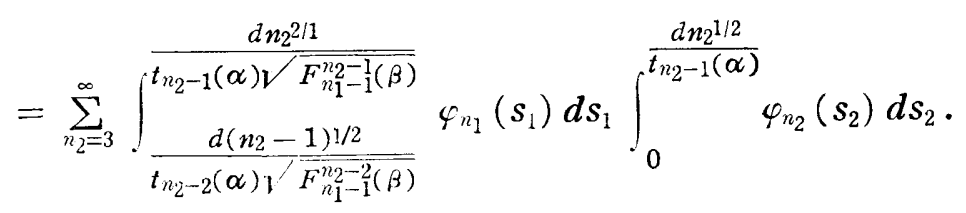

\section{$\S 2$. Numerical considerations.}

Each term of the sums of the integrals in the last right hand side of (1.10) may be caluculated by means of the tables of the incomplete $I$ function [1]. We have constructed the distributoin functions of the stochastic variables $\boldsymbol{n}_{2}$ with respect to the set of the values of $\alpha, \beta, d^{2} \sigma^{-2}$ and $\boldsymbol{n}_{1}$, where $\alpha=0.01,0.05 ; \beta=0.01,0.05 ; d^{2} \sigma^{-2}=0.75,0.25 ; \quad n_{1}=5,10,15,21,25,31$. The results are given in Tables I, II, III and IV. These tables suggest us the way how to choose a sample size $n_{2}$ in our two sample theoretical formulations; indeed this choose will be seen to depend upon the values of $\alpha$, $\beta$ and $d^{2} \sigma^{-2}$.

\section{§ 3. General remarks.}

There remaines the problem of two dependent samples. That is to say, in stead of taking consideration a new independent second sample $O_{2}$, we may consider a pooled sample of size $\boldsymbol{n}_{1}+\boldsymbol{n}_{2}$, adding a new sample of size $\boldsymbol{n}_{2}$ to that of size $\boldsymbol{n}_{1}$. This problem was considered by MoOD [1], but, as he himself pointed out in MooD [2], there was an error because he had been in failing to take account of the fact that $\boldsymbol{n}$ is a random variable.

In these problems there may be various procedures which will give us two sample formulations. The method due to STEIN [1] is specially worth while to mention, and KITAGAwA [1] gave some stochastic considerations about similar problems in Part III, and Kitagawa [4] applies Stein-Barnard method to BEHRENS-FISHER's test. The two dependent sample formulations will be reconsidered in another occassion.

\section{References}

Marilyn Harris, D. G. Worvitz and A. M. Mood. [1]: On the determination of sample sizes in designing experiments, Jour. Ame. Atat. Associ., sep. 1948.

A. M. Mood ; Erratum [2]: On the determination of sample size in designing experiments, Jour. Ameri. Stat. Associ., December 1951, vol. 46, No. 256.

Karl Pearson,F.R.S. [1]: Tables of the incomplete $\Gamma$-function, 1922, London.

Charles Stein. [1]: A Two Sample Test for a lineear Hypothesis whose Power is Independent of the Variance, Ann. Math. Stat., vol. 15, 1945, p. 234.

Kitagawa, T. [1]: Successive process of statistical inferences, Mem. Fac. Sci., Ser. A, 5 $(1950), 139-180$.

KItagawa, T. [2]: Successive process of statistical inferences, Mem. Fac. Sci., Ser. A, 6 (1951), $55-95$.

KiKagawa, T. [3]: Successive process of statistical inferences, Man. Fac. Sci., Ser. A, 7 (1952) , $131-155$.

Kitagawa, T. [4]: Successive process of statistical inferences, Bull. Math. Stat. Resrech Association of Stat. Sci, vol. 5, No. 1-2. 
Table of the Individual probabilities:

$$
\begin{aligned}
I\left(n_{2} ; n_{1} / d^{2} \sigma-2, \alpha, \beta\right) \\
=\int_{b\left(n_{2}-1\right)}^{b\left(n_{2}\right)} \varphi_{n_{1}-1}\left(s_{1} ; \sigma\right) d s_{1} \int_{0}^{c\left(n_{\Sigma}\right)} \varphi_{n_{2}}\left(s_{2} ; \sigma\right) d s_{2} \\
b(n) \equiv d n^{1 / 2}\left\{t_{3 i-1}(\alpha)\right\}^{-1}\left\{F_{n_{1}-1}^{n-1}(\beta)\right\}^{-1 / 2} \\
c\left(n_{2}\right) \equiv d n_{2} 1 / 2\left\{t_{n_{2}-1}(\alpha)\right\}^{-1}
\end{aligned}
$$

\begin{tabular}{|c|c|c|c|c|c|c|c|c|c|c|c|}
\hline$n_{1} n_{1}$ & 11 & 12 & 13 & 14 & 15 & 16 & 17 & 18 & 19 & 20 & C.S. \\
\hline 21 & 0 & 0.0002 & 0.0008 & 0.0017 & 0.0034 & 0.0067 & 0.0105 & 0.0155 & 0.0219 & 0.0292 & 0.0899 \\
\hline 25 & 0 & 0.0001 & 0.0005 & 0.0014 & 0.0026 & 0.0073 & 0.0106 & 0.0181 & 0.0258 & 0.0350 & 0.1014 \\
\hline 31 & 0 & 0.0001 & 0.0002 & 0.0009 & 0.6027 & 0.0061 & 0.0135 & 0.0199 & 0.0309 & 0.0438 & 0.1181 \\
\hline$n_{1} n_{\varepsilon}$ & 21 & 22 & 23 & 24 & 25 & 26 & 27 & 28 & 29 & 30 & C.S. \\
\hline 21 & 0.0362 & 0.0416 & 0.0475 & 0.0524 & 0.0568 & 0.0568 & 0.0582 & 0.0572 & 0.0565 & 0.0551 & 0.6082 \\
\hline 25 & 0.0456 & 0.0524 & 0.0577 & 0.0665 & 0.0680 & 0.0676 & 0.0670 & 0.0657 & 0.0593 & 0.0574 & 0.7086 \\
\hline 31 & 0.0579 & 0.0610 & 0.0807 & 0.0818 & 0.0842 & 0.0790 & 0.0749 & 0.0682 & 0.0605 & 0.0525 & 0.8188 \\
\hline$n_{1} n^{n_{2}}$ & 31 & 32 & 33 & 34 & 35 & 36 & 37 & 38 & 39 & 40 & C.S. \\
\hline 21 & 0.0504 & 0.0439 & 0.0425 & 0.0354 & 0.0330 & 0.0297 & 0.0255 & 0.0224 & 0.0189 & 0.0162 & 0.9261 \\
\hline 25 & 0.0495 & 0.0413 & 0.0359 & 0.0310 & 0.0263 & 0.0216 & 0.0179 & 0.0144 & 0.0114 & 0.0089 & 0.9668 \\
\hline 31 & 0.0422 & 0.0322 & 0.0259 & 0.0203 & 0.0157 & 0.0116 & 0.0087 & 0.0062 & 0.0043 & 0.0030 & 0.9889 \\
\hline$n_{1} n_{2}$ & 41 & 42 & 43 & 44 & 45 & 46 & 47 & 48 & 49 & 50 & C.S. \\
\hline 21 & 0.0133 & 0.0100 & 0.0084 & 0.0068 & 0.0057 & 0.0045 & 0.0038 & 0.0029 & 0.0024 & 0.0019 & 0.9858 \\
\hline 25 & 0.0099 & 0.0047 & 0.0037 & 0.0027 & 0.0021 & 0.0016 & 0.0012 & 0.0008 & 0.0007 & 0.0004 & 0.9946 \\
\hline 31 & 0.0021 & 0.0012 & 0.0009 & 0.0005 & 0.0004 & 0.0003 & 0.0001 & 0.0001 & 0.0001 & 0 & 0.9946 \\
\hline$n_{1} n_{2}$ & 51 & 52 & 53 & 54 & 55 & 56 & 57 & 58 & 59 & 60 & T.S. \\
\hline 21 & 0.0014 & 0.0008 & 0.0009 & 0.0007 & 0.0005 & 0.0004 & 0.0003 & 0.0002 & 0.0002 & 0.0002 & 0.9914 \\
\hline 25 & 0.0003 & 0.0001 & 0.0001 & 0.0001 & 0.0001 & 0.0001 & 0 & & & & 0.9954 \\
\hline 31 & 0 & & & & & & & & & & 0.9946 \\
\hline
\end{tabular}

Table I-(1) $\quad\left(\alpha=\beta=1 \% ; d^{2} \sigma-2=0.75\right)$ 
Table I-(2) $\quad\left(\alpha=\beta=1 \% ; d^{2} \sigma^{-2}=0.5\right)$

\begin{tabular}{|c|c|c|c|c|c|c|c|c|c|c|c|}
\hline$n_{1} \quad n_{2}$ & 11 & 12 & 13 & 14 & 15 & 16 & 17 & 18 & 19 & 20 & C.S. \\
\hline 25 & 0 & 0 & 0 & 0 & 0 & 0.0002 & 0.0004 & 0.0025 & 0.0015 & 0.0028 & 0.0074 \\
\hline 31 & 0 & 0 & 0 & 0 & 0 & 0.0001 & 0.0002 & 0.0006 & 0.0011 & 0.0026 & 0.0046 \\
\hline$n_{1} \quad n_{2}$ & 21 & 22 & 23 & 24 & 25 & 26 & 27 & 28 & 29 & 30 & C.S. \\
\hline 25 & 0.0047 & 0.0085 & $0.009 \mathrm{i}$ & 0.0133 & 0.0171 & 0.0208 & 0.0244 & 0.0299 & 0.0339 & 0.0384 & 0.2075 \\
\hline 31 & 0.0045 & 0.0067 & 0.0110 & 0.0154 & 0.0219 & 0.0262 & 0.0319 & 0.0379 & 0.0442 & 0.0499 & 0.2562 \\
\hline $\bar{n}_{1} n_{n_{1}}$ & 31 & 32 & 33 & 34 & 35 & 36 & 37 & 38 & 39 & 40 & C.S. \\
\hline 25 & 0.0407 & 0.0410 & 0.0432 & 0.0451 & 0.0463 & 0.0466 & 0.0464 & 0.0459 & 0.0443 & 0.0427 & 0.6497 \\
\hline 31 & 0.0526 & 0.0524 & 0.0543 & 0.0551 & 0.0550 & 0.0537 & 0.0521 & 0.0492 & 0.0454 & 0.0418 & 0.7658 \\
\hline$n_{1} n_{2}$ & 41 & 42 & 43 & 44 & 45 & 46 & 47 & 48 & 49 & 50 & C.S. \\
\hline 25 & 0.0406 & 0.0316 & 0.0338 & 0.0290 & 0.0267 & 0.0241 & 0.0219 & 0.0196 & 0.0173 & 0.0154 & 0.9097 \\
\hline 31 & 0.0375 & 0.0296 & 0.0263 & 0.0228 & 0.0197 & 0.0170 & 0.0142 & 0.0120 & 0.0099 & 0.0072 & 0.9620 \\
\hline$n_{1} \quad n_{2}$ & 51 & 52 & 53 & 54 & 55 & 56 & 57 & 58 & 59 & 60 & T.S. \\
\hline 25 & 0.0133 & 0.0113 & 0.0102 & 0.0087 & 0.0073 & 0.0063 & 0.0052 & 0.0043 & 0.0036 & 0.0031 & 0.9840 \\
\hline 31 & 0.0065 & 0.0051 & 0.0044 & 0.0034 & 0.0026 & 0.0021 & 0.0016 & 0.0012 & 0.0009 & 0.0007 & 0.9905 \\
\hline
\end{tabular}


Table II - (1) $\quad\left(\alpha=1 \%, \beta=5 \% ; d^{2} \sigma^{-2}=0.75\right)$

\begin{tabular}{|c|c|c|c|c|c|c|c|c|c|c|c|c|}
\hline \begin{tabular}{ll|}
$n_{1}$ & $n_{2}$ \\
\end{tabular} & 7 & 8 & 9 & 10 & 11 & 12 & 13 & 14 & 15 & 16 & 17 & C.s. \\
\hline 10 & 0 & 0.0002 & 0.0005 & 0.0014 & 0.0027 & 0.0048 & 0.0077 & 0.0111 & $|0.0157|$ & 0.0203 & 0.0245 & 0.0889 \\
\hline 15 & 0 & 0 & 0 & 0.0009 & 0.0018 & 0.0039 & 0.0074 & 0.0128 & 0.0196 & 0.0273 & 0.0342 & 0.1078 \\
\hline 21 & 0 & 0 & 0 & 0.0001 & 0.0009 & 0.0027 & 0.0064 & 0.0117 & $|0.0212|$ & $0.0340 \mid$ & $|0.0445|$ & 0.1215 \\
\hline 25 & 0 & 0 & 0 & 0 & 0.0005 & 0.0019 & $|0.0054|$ & $|0.0116|$ & $0.0221 \mid$ & $0.0362 \mid$ & 0.0500 & 0.1277 \\
\hline 31 & 0 & 0 & 0 & 0 & 0.0003 & 0.0012 & 0.0042 & 0.0105 & 0.0219 & 0.0396 & 0.0569 & 0.1346 \\
\hline$n_{1} \quad n_{2}$ & 18 & 19 & 20 & 21 & 22 & 23 & 24 & 25 & 26 & 27 & 28 & c.s. \\
\hline 10 & 0.0280 & 0.0311 & 0.0337 & $0.0360 \mid$ & 0.0368 & 0.0378 & 0.0386 & 0.0385 & 0.0377 & 0.0372 & 0.0362 & 0.4805 \\
\hline 15 & $|0.0419|$ & $|0.0481|$ & $|0.0520|$ & $0.0549 \mid$ & 0.0567 & 0.0571 & 0.0564 & 0.0547 & 0.0505 & 0.0474 & 0.0438 & 0.0613 \\
\hline 21 & $|0.0554|$ & $|0.0661|$ & $0.0734 \mid$ & $0.0756 \mid$ & 0.0740 & 0.0716 & 0.0684 & 0.0619 & 0.0536 & 0.0466 & 0.0395 & 0.8076 \\
\hline 25 & 0.0646 & 0.0770 & 0.0851 & 0.0870 & 0.0830 & $|0.0789|$ & $|0.0715|$ & 0.0626 & $|0.0507|$ & 0.0413 & 0.0330 & 0.8624 \\
\hline 31 & 0.0767 & 0.0921 & 0.0998 & 0.1068 & 0.0935 & 0.0842 & 0.0713 & 0.0580 & $0.0366 \mid$ & $0.0287 \mid$ & 0.0236 & 0.9059 \\
\hline$n_{1} \quad n_{2}$ & 29 & 30 & 31 & 32 & 33 & 34 & 35 & 36 & 37 & 38 & 39 & C.S. \\
\hline 10 & 0.0350 & 0.0330 & 0.0320 & $0.0296 \mid$ & 0.0283 & 0.0270 & 0.0256 & $0.0242 \mid$ & $0.0225 \mid$ & 0.0210 & 0.0196 & 0.7783 \\
\hline 15 & 0.0397 & 0.0350 & 0.0313 & 0.0268 & 0.0238 & 0.0207 & 0.0170 & 0.0147 & 0.0131 & 0.0112 & 0.0094 & 0.9140 \\
\hline 21 & $|0.0326|$ & 0.0272 & $\mid 0.0211$ & 0.0160 & 0.0128 & 0.0099 & 0.0076 & $|0.0058|$ & 0.0043 & 0.0032 & 0.0024 & 0.9505 \\
\hline 25 & 0.0259 & 0.0199 & 0.0143 & 0.0100 & 0.0073 & 0.0052 & $\mid 0.0037$ & 0.0025 & 0.0017 & 0.0011 & 0.0007 & 0.9547 \\
\hline 31 & 0.0166 & 0.0166 & 0.0073 & 0.0044 & 0.0029 & $0.0017 \mid$ & 0.0011 & 0.0006 & $0.0003 \mid$ & $0.0001 \mid$ & 0 & 0.9525 \\
\hline$n_{1} n_{\varepsilon}$ & 40 & 41 & 42 & 43 & 44 & 45 & 46 & 47 & 48 & 49 & 50 & C S. \\
\hline 10 & $|0.0181|$ & $0.0168 \mid$ & $|0.0142|$ & 0.0129 & $0.0120 \mid$ & $|0.0110|$ & 0.0100 & 0.0090 & 0.0083 & 0.0075 & 0.0062 & 0.9043 \\
\hline 15 & 0.0078 & 0.0060 & 0.0051 & 0.0041 & 0.0033 & 0.0028 & 0.0022 & 0.0018 & 0.0015 & 0.0012 & 0.0009 & 0.9507 \\
\hline 21 & 0.0017 & 0.0012 & 0.0007 & 0.0006 & 0.0004 & 0.0003 & 0.0002 & 0.0001 & 0.0001 & $0.0001 \mid$ & 0 & 0.9559 \\
\hline 25 & 0.0003 & 0.0003 & 0.0001 & 0 & & & & & & & & 0.9554 \\
\hline 31 & 0 & & & & & & & & & & & 0.9525 \\
\hline$n_{1} \quad n_{2}$ & 51 & 52 & 53 & 54 & 55 & 56 & 57 & 58 & 59 & 60 & & T.S. \\
\hline 10 & 0.0057 & $|0.0053|$ & $0.0051 \mid$ & $0.0044 \mid$ & 0.0039 & $0.0035 \mid$ & $0.0032 \mid$ & 0.0028 & $0.0024 \mid$ & 0.0022 & & 0.9423 \\
\hline 15 & 0.0008 & 0.0005 & 0.0005 & 0.0004 & 0.0003 & $|0.0002|$ & $|0.0002|$ & 0.0001 & $|0.0001|$ & 0.0001 & & 0.09539 \\
\hline 21 & 0 & & & & & & & & & & & 0.9559 \\
\hline 25 & 0 & & & & & & & & & & & 0.9554 \\
\hline 31 & 0 & & & & & & & & & & & 0.9525 \\
\hline
\end{tabular}


Table II - (2) $\quad\left(\alpha=1 \%, \beta=5 \% ; d^{2} \sigma-2=0.5\right)$

\begin{tabular}{|c|c|c|c|c|c|c|c|c|c|c|c|c|}
\hline$n_{1} \quad n_{2}$ & 12 & 13 & 14 & 15 & 16 & 17 & 18 & 19 & 20 & 21 & 22 & C.S. \\
\hline 21 & 0.0001 & 0.0002 & 0.0004 & 0.0011 & 0.0024 & 0.0042 & 0.0069 & 0.0107 & 0.0157 & 0.0219 & 0.0269 & 0.0905 \\
\hline 25 & 0 & 0 & $0.0002 \mid$ & 0.0007 & 0.0018 & 0.0036 & 0.0063 & 0.0107 & 0.0164 & 0.0235 & 0.0302 & 0.0934 \\
\hline 31 & 0 & 0 & 0.0001 & 0.0004 & 0.0012 & 0.0028 & 0.0056 & 0.0105 & 0.0170 & 0.0254 & 0.0357 & 0.0987 \\
\hline$n_{1} n^{n_{s}}$ & 23 & 24 & 25 & 26 & 27 & 28 & 29 & 30 & 31 & 32 & 33 & C.S. \\
\hline 21 & 0.0327 & 0.0383 & 0.0441 & 0.0465 & 0.0495 & 0.0512 & 0.0522 & 0.0520 & 0.0503 & 0.0480 & 00444 & 0.5992 \\
\hline 25 & 0.0372 & $0.0454 \mid$ & 0.0513 & 0.0543 & 0.0575 & 0.0594 & 0.0608 & $|0.0592|$ & 0.0551 & $0.0492 \mid$ & $\mid 0.0466$ & 0.6694 \\
\hline 31 & 0.0441 & 0.0534 & 0.0618 & 0.0652 & 0.0685 & 0.0694 & 0.0680 & 0.0660 & 0.0593 & 0.0489 & 0.0455 & 0.7488 \\
\hline$n_{1} n_{2}$ & 34 & 35 & 36 & 37 & 38 & 39 & 40 & 41 & 42 & 43 & 44 & C.S. \\
\hline 21 & 0.0421 & $|0.0390|$ & 0.0357 & 0.0332 & 0.0302 & 0.0271 & 0.0230 & 0.0194 & 0.0166 & 0.0146 & 0.0124 & 0.8930 \\
\hline 25 & $0.0419 \mid$ & 0.0384 & 0.0343 & 0.0301 & 0.0262 & 0.0224 & 0.0188 & $\mid 0.0158$ & $\mid 0.0118$ & 0.0097 & 0.0080 & 0.9268 \\
\hline 31 & 0.0400 & 0.0344 & 0.0287 & 0.0238 & 0.0193 & 0.0154 & 0.0120 & 0.0088 & 0.0066 & 0.0051 & 0.0036 & 0.9465 \\
\hline$n_{1} \quad n_{2}$ & 45 & 46 & 47 & 48 & 49 & 50 & 51 & 52 & 53 & 54 & 55 & C.S. \\
\hline 21 & 0.0107 & 0.0091 & 0.0079 & 0.0064 & 0.0054 & 0.0046 & 0.0037 & 0.0030 & 0.0024 & 0.0020 & 0.0017 & 0.9499 \\
\hline 25 & 0.0065 & 0.0052 & 0.0041 & 0.0033 & 0.0026 & $0.0020 \mid$ & 0.0015 & $0.0012 \mid$ & 0.0008 & 0.0006 & 0.0005 & 0.9551 \\
\hline 31 & 0.0027 & 0.0020 & 0.0015 & 0.0010 & 0.0007 & 0.0004 & 0.0003 & 0.0002 & 0.0001 & 0 & & 0.9554 \\
\hline$n_{1} \quad u_{2}$ & 56 & 57 & 58 & 59 & 60 & & & & & & & T.S. \\
\hline 21 & 0.0014 & 0.0011 & 0.0008 & 0.0006 & 0.6004 & & & & & & & 0.9542 \\
\hline 25 & 0.0004 & 0.0003 & 0.0001 & 0.0001 & 0 & & & & & & & 0.9560 \\
\hline 31 & & & & & & & & & & & & 0.9554 \\
\hline
\end{tabular}


Tab1e III - (1) $\quad\left(\alpha=5 \%, \beta=1 \% ; d^{2} \sigma^{-2}=0.75\right)$

\begin{tabular}{|c|c|c|c|c|c|c|c|c|c|c|c|}
\hline $\begin{array}{ll}n_{2} & n_{1} \\
& \end{array}$ & 6 & 7 & 8 & 9 & 10 & 11 & 12 & 13 & 14 & 15 & c.s. \\
\hline 15 & 0 & 0.0005 & 0.0011 & 0.0032 & 0.0077 & 0.0143 & 0.0231 & 0.0336 & 0.0424 & c.0528 & 0.1787 \\
\hline 21 & 0 & 0 & 0.0005 & 0.0022 & 0.0070 & 0.0171 & 0.0301 & 0.0489 & 0.0655 & 0.0821 & 0.2534 \\
\hline 25 & 0 & 0 & 0.0002 & 0.0016 & 0.0058 & 0.0169 & 0.0336 & 0.0591 & 0.0803 & 0.1008 & 0.2983 \\
\hline 31 & 0 & 0 & 0.0001 & 0.0010 & 0.0048 & 0.0160 & 0.0373 & 0.0697 & 0.0998 & 0.1262 & 0.3549 \\
\hline$n_{1} \quad n_{2}$ & 16 & 17 & 18 & 19 & 20 & 21 & 22 & 23 & 24 & 25 & c.s. \\
\hline 15 & 0.0611 & 0.0642 & 0.0678 & 0.0695 & 0.0690 & $0.0667 \mid$ & 0.0600 & 0.0548 & 0.0496 & 0.0443 & 0.7857 \\
\hline 21 & 0.0950 & 0.0951 & 0.0949 & 0.0899 & 0.0807 & 0.0699 & 0.0543 & 0.0326 & 0.0449 & 0.0255 & 0.9362 \\
\hline 25 & 0.1147 & 0.1105 & 0.1052 & 0.0935 & 0.0780 & 0.0613 & 0.0435 & 0.0314 & 0.0217 & 0.0146 & 0.9727 \\
\hline 31 & 0.1393 & 0.1262 & 0.1106 & 0.0911 & 0.0654 & 0.0449 & 0.0269 & 0.0166 & 0.0095 & 0.0053 & 0.9907 \\
\hline $\bar{n}_{1} n_{2}$ & 26 & 27 & 28 & 29 & 30 & 31 & 32 & 33 & 34 & 35 & C.S. \\
\hline 15 & 0.0368 & 0.0319 & 0.0271 & 0.0224 & 0.0188 & 0.0153 & 0.0116 & 0.0096 & 0.0077 & 0.0060 & 0.9729 \\
\hline 21 & 0.0181 & 0.0128 & 0.0092 & 0.0062 & 0.0043 & 0.0028 & 0.0017 & 0.0011 & 0.0008 & 0.0005 & 0.9937 \\
\hline 25 & 0.0090 & 0.0056 & 0.0035 & 0.0021 & 0.0012 & 0.0007 & 0.0004 & 0.0002 & 0.0001 & 0 & 0.9955 \\
\hline 31 & 0.0026 & 0.0007 & 0.0007 & 0.0003 & 0.0002 & 0.0001 & 0 & 0 & 0 & 0 & 0.9953 \\
\hline${ }_{n_{1}} n_{2}$ & 36 & 37 & 38 & 39 & 40 & 41 & 42 & 43 & 44 & 45 & c.S. \\
\hline 15 & 0.0048 & 0.0038 & 0.0029 & 0.0023 & 0.0017 & 0.0014 & 0.0008 & 0.0007 & 0.0006 & 0.0003 & 0.9922 \\
\hline 21 & 0.0002 & 0.0002 & 0.0001 & 0.0001 & 0 & 0 & & & & & 0.9943 \\
\hline 25 & 0 & & & & & & & & & & - \\
\hline 31 & 0 & & & & & & & & & & - \\
\hline$n_{n_{1}} n_{2}$ & 46 & 47 & 48 & 49 & 50 & 51 & 52 & 53 & 54 & 55 & T.S. \\
\hline 15 & 0.0004 & 0.0002 & 0.0002 & 0.0001 & 0.0001 & 0 & & & & & 0.9932 \\
\hline 21 & & & & & & & & & & & 0.9943 \\
\hline 25 & & & & & & & & & & & 0.9955 \\
\hline 31 & & & & & & & & & & & 0.9953 \\
\hline
\end{tabular}


Table III $-(2) \quad\left(\alpha=5 \%, \beta=1 \% ; d^{2} \sigma^{-2}=0.5\right)$

\begin{tabular}{|c|c|c|c|c|c|c|c|c|c|c|c|}
\hline$n_{1} n_{2}$ & 9 & 10 & 11 & 12 & 13 & 14 & 15 & 16 & 17 & 18 & C.S. \\
\hline 21 & 0 & 0.0003 & 0.0010 & 0.0024 & 0.0055 & 0.0091 & 0.0163 & 0.0242 & 0.0322 & 0.0405 & 0.1315 \\
\hline 25 & 0 & 0.0002 & 0.0007 & 0.0019 & 0.0050 & 0.0095 & 0.0175 & 0.0282 & 0.0382 & 0.0502 & 0.1514 \\
\hline 31 & 0 & 0.0001 & 0.0004 & 0.0031 & 0.0041 & 0.0091 & 0.0188 & 0.0334 & 0.0469 & 0.0631 & 0.1772 \\
\hline $\begin{array}{lll} & n_{2} \\
& & \\
\end{array}$ & 19 & 20 & 21 & 22 & 23 & 24 & 25 & 26 & 27 & 28 & c.s. \\
\hline 21 & 0.0498 & 0.0576 & 0.0641 & 0.0651 & 0.0667 & 0.0670 & 0.0652 & 0.0596 & 0.0551 & 0.0502 & 0.7319 \\
\hline 25 & 0.0615 & 0.0715 & 0.0786 & 0.0777 & 0.0776 & 0.0751 & 0.0701 & 0.0608 & 0.0534 & 0.0458 & 0.8235 \\
\hline 31 & 0.0774 & 0.0905 & 0.0954 & 0.0924 & 0.0875 & 0.0800 & 0.0697 & 0.0549 & 0.0445 & 0.0348 & 0.9043 \\
\hline$n_{1} n_{2}$ & 29 & 30 & 31 & 32 & 33 & 34 & 35 & 86 & 37 & 38 & C.S. \\
\hline 21 & 0.0450 & 0.0392 & 0.0339 & 0.0272 & 0.0229 & 0.0193 & 0.0157 & 0.0128 & 0.0104 & 0.0081 & 0.9694 \\
\hline 25 & 0.0382 & 0.0314 & 0.0251 & 0.0185 & 0.0145 & 0.0112 & 0.0084 & 0.0064 & 0.0047 & 0.0033 & 0.9852 \\
\hline 31 & 0.0260 & 0.0192 & 0.0136 & 0.0088 & 0.0062 & 0.0041 & 0.0028 & 0.0018 & 0.0011 & 0.0007 & 0.9886 \\
\hline$n_{n_{1}} n^{n_{s}}$ & 39 & 40 & 41 & 42 & 43 & 44 & 45 & 46 & 47 & 48 & C.S. \\
\hline 21 & 0.0065 & 0.0040 & 0.0039 & 0.0026 & 0.0021 & 0.0016 & 0.0012 & 0.0009 & 0.0007 & 0.0005 & 0.9904 \\
\hline 25 & 0.0024 & 0.0019 & 0.0009 & 0.0007 & 0.0005 & 0.0004 & 0.0002 & 0.0002 & 0.0001 & 0.0001 & 0.9926 \\
\hline 31 & 0.0004 & 0.0003 & 0.0001 & 0.0001 & 0 & 0 & 0 & 0 & 0 & 0 & 0.9895 \\
\hline$n_{1} \quad n_{2}$ & 49 & 50 & 51 & 52 & 53 & 54 & 55 & 56 & 57 & 59 & T.S. \\
\hline 21 & 0.0003 & 0.0003 & 0.0002 & 0.0002 & 0.0001 & 0 & & & & & 0.9915 \\
\hline 25 & 0 & 0 & & & & & & & & & 0.9926 \\
\hline 31 & 0 & 0 & & & & & & & & & 0.9895 \\
\hline
\end{tabular}

Table III - (3) $\quad\left(\alpha=5 \%, \beta=1 \% ; d^{2} / \sigma^{2}=0.25\right)$

\begin{tabular}{|c|c|c|c|c|c|c|c|c|c|c|c|}
\hline $\boldsymbol{n}_{1} \quad \boldsymbol{n}_{2}$ & 16 & 17 & 18 & 19 & 20 & 21 & 22 & 23 & 24 & 25 & C.S. \\
\hline 31 & 0 & 0.0001 & 0.0003 & 0.0006 & 0.0012 & 0.0023 & 0.0036 & 0.0055 & 0.0083 & 0.0114 & 0.0333 \\
\hline $\begin{array}{ll} & n_{2} \\
n_{1} & \\
\end{array}$ & 26 & 27 & 28 & 29 & 30 & 31 & 32 & 33 & 34 & 35 & C.S. \\
\hline 31 & 0.0154 & 0.0194 & 0.0239 & 0.0285 & 0.0337 & 0.0384 & 0.0415 & 0.0440 & 0.0462 & 0.0478 & 0.3721 \\
\hline$n_{1} \quad n_{2}$ & 36 & 37 & 38 & 39 & 40 & 41 & 42 & 43 & 44 & 45 & C.S. \\
\hline 31 & 0.0491 & 0.0498 & 0.0496 & 0.0489 & 0.0475 & 0.0447 & 0.0384 & 0.0359 & 0.0332 & 0.0305 & 0.7997 \\
\hline$n_{1} n_{2}$ & 46 & 47 & 48 & 49 & 50 & 51 & 52 & 53 & 54 & 55 & C.S. \\
\hline 31. & 0.0272 & 0.0245 & 0.0218 & 0.0193 & 0.0170 & 0.0144 & 0.0124 & 0.0107 & 0.0090 & 0.0088 & 0.9648 \\
\hline $\begin{array}{ll} & n_{2} \\
n_{1} & \end{array}$ & 56 & 57 & 58 & 59 & 60 & 61 & 62 & 63 & 64 & 65 & T.S. \\
\hline 31 & 0.0050 & 0.0051 & 0.0044 & 0.0037 & 0.0028 & 0.9858 & & & & & \\
\hline
\end{tabular}


Table IV - (2) $\quad\left(\alpha=\beta=5 \% ; d^{2} \sigma-2=0.5\right)$

\begin{tabular}{r|c|c|c|c|c|c|c|c|c|c|c|c}
\hline$n_{1} n_{2}$ & 3 & 4 & 5 & 6 & 7 & 8 & 9 & 10 & 11 & 12 & C.S. \\
\hline 10 & & & 0 & 0.0002 & 0.0008 & 0.0016 & 0.0034 & 0.0063 & 0.0110 & 0.0159 & 0.0392 \\
\hline 15 & & & & 0 & 0.0002 & 0.0006 & 0.0021 & 0.0051 & 0.0104 & 0.0176 & 0.0360 \\
\hline 21 & & & & & 0 & 0.0002 & 0.0011 & 0.0034 & 0.0087 & 0.0153 & 0.0287 \\
\hline$n_{1}$ & 13 & 14 & 15 & 16 & 17 & 18 & 19 & 20 & 21 & 22 & C.S. \\
\hline 10 & 0.0207 & 0.0253 & 0.0315 & 0.0362 & 0.0390 & 0.0413 & 0.0433 & 0.0443 & 0.0450 & 0.0448 & 0.4106 \\
\hline 15 & 0.0274 & 0.0355 & 0.0469 & 0.0557 & 0.0602 & 0.0642 & 0.0660 & 0.0657 & 0.0646 & 0.0588 & 0.5810 \\
\hline 21 & 0.0362 & 0.0390 & 0.0615 & 0.0730 & 0.0823 & 0.0865 & 0.0862 & 0.0820 & 0.0752 & 0.0633 & 0.7139 \\
\hline$n_{2}$ & 23 & 24 & 25 & 26 & 27 & 28 & 29 & 30 & 31 & 32 & C.S. \\
\hline 10 & 0.0439 & 0.0418 & 0.0397 & 0.0377 & 0.0358 & 0.0338 & 0.0315 & 0.0295 & 0.0271 & 0.0243 & 0.7557 \\
\hline 15 & 0.0546 & 0.0496 & 0.0449 & 0.0383 & 0.0335 & 0.0288 & 0.0244 & 0.0207 & 0.0170 & 0.0140 & 0.9068 \\
\hline 21 & 0.0538 & 0.0445 & 0.0352 & 0.0282 & 0.0213 & 0.0161 & 0.0119 & 0.0087 & 0.0062 & 0.0043 & 0.9439 \\
\hline 15 & 0.0111 & 0.0092 & 0.0074 & 0.0059 & 0.0048 & 0.0038 & 0.0028 & 0.0025 & 0.0017 & & 0.9560 \\
\hline 21 & 0.0030 & 0.0021 & 0.0014 & 0.0010 & 0.0007 & 0.0004 & 0.0003 & 0.0002 & 0.0001 & & 0.9531 \\
\hline$n_{1}$ & 33 & 34 & 35 & 36 & 37 & 38 & 39 & 40 & 41 & & T.S. \\
\hline 10 & 0.0246 & 0.0205 & 0.0186 & 0.0169 & 0.0153 & 0.0139 & 0.0126 & 0.0117 & & & 0.8898 \\
\hline
\end{tabular}


Table IV - (3) $\quad\left(\alpha=\beta=5 \% ; d^{2} \sigma^{-2}=0.25\right)$

\begin{tabular}{|c|c|c|c|c|c|c|c|c|c|c|c|}
\hline$n_{1} \quad n_{2}$ & 8 & 9 & 10 & 11 & 12 & 13 & 14 & 15 & 16 & 17 & C.S. \\
\hline 10 & & 0 & 0.0001 & 0.0002 & 0.0006 & 0.0005 & 0.0011 & 0.0017 & 0.0024 & 0.0033 & 0.0099 \\
\hline 15 & & & 0 & 0.0001 & 0.0002 & 0.0004 & 0.0007 & 0.0013 & 0.0021 & 0.0031 & 0.0079 \\
\hline 21 & & & & 0 & 0.0001 & 0.0002 & 0.0005 & 0.0010 & 0.0017 & 0.0026 & 0.0061 \\
\hline$n_{n_{1}} n_{2}$ & 18 & 19 & 20 & 21 & 22 & 23 & 24 & 25 & 26 & 27 & C.S. \\
\hline 10 & 0.0045 & 0.0060 & 0.0073 & 0.0092 & 0.0110 & 0.0129 & 0.0140 & 0.0150 & 0.0163 & 0.0177 & 0.1238 \\
\hline 15 & 0.0045 & 0.0069 & 0.0092 & 0.0118 & 0.0142 & 0.0170 & 0.0200 & 0.0233 & 0.0252 & 0.0273 & 0.1673 \\
\hline 21 & 0.0042 & 0.0067 & 0.0098 & 0.0137 & 0.0176 & 0.0223 & 0.0274 & 0.0308 & 0.0356 & 0.0383 & 0.2125 \\
\hline $\begin{array}{ll}n_{1} & n_{2} \\
\end{array}$ & 28 & 29 & 30 & 31 & 32 & 33 & 34 & 35 & 36 & 37 & c.s. \\
\hline 10 & 0.0185 & 0.0196 & 0.0202 & 0.0210 & 0.0214 & 0.0216 & 0.0218 & 0.0220 & 0.0223 & 0.0224 & 0.3346 \\
\hline 15 & 0.0296 & 0.0305 & 0.0314 & 0.0317 & 0.0324 & 0.0330 & 0.0333 & 0.0331 & 0.0330 & 0.0326 & 0.4879 \\
\hline 21 & 0.0418 & 0.0430 & 0.0452 & 0.0459 & 0.0448 & 0.0435 & 0.0428 & 0.0414 & 0.0397 & 0.0375 & 0.6381 \\
\hline \begin{tabular}{ll|} 
& $n_{2}$ \\
$n_{1}$ & \\
\end{tabular} & 38 & 39 & 40 & 41 & 42 & 43 & 44 & 45 & 46 & 47 & c.s. \\
\hline 10 & 0.0225 & 0.0224 & 0.0223 & 0.0220 & 0.0213 & 0.0208 & 0.0203 & 0.0201 & 0.0196 & 0.0193 & 0.5452 \\
\hline 15 & 0.0320 & 0.0312 & 0.0307 & 0.0291 & 0.0270 & 0.0257 & 0.0234 & 0.0223 & 0.0211 & 0.0199 & 0.7503 \\
\hline 21 & 0.0354 & 0.0330 & 0.0300 & 0.0270 & 0.0241 & 0.0217 & 0.0196 & 0.0174 & 0.0154 & 0.0138 & 0.8755 \\
\hline \begin{tabular}{ll|} 
& $n_{2}$ \\
$n_{1}$ & \\
\end{tabular} & 48 & 49 & 50 & 51 & 52 & 53 & 54 & 55 & 56 & 57 & c.s. \\
\hline 10 & 0.0188 & 00.185 & 0.0180 & 0.0177 & 0.0170 & 0.0165 & 0.0160 & 0.0156 & 0.0150 & 0.0144 & 0.7127 \\
\hline 15 & 0.0183 & 0.0172 & 0.0161 & 0.0150 & 0.0138 & 0.0125 & 0.0116 & 0.0107 & 0.0098 & 0.0089 & 0.8842 \\
\hline 21 & 0.0122 & 0.0106 & 0.0092 & 00.081 & 0.0070 & 0.0050 & 0.0051 & 0.0043 & 0.0036 & 0.0030 & 0.9446 \\
\hline$n_{n_{1}} n_{2}$ & 58 & 59 & 60 & Total & & & & & & & \\
\hline 10 & 0.0136 & 0.0133 & 0.0120 & 0.7516 & & & & & & & \\
\hline 15 & 0.0080 & 0.0070 & 0.0061 & 0.9053 & & & & & & & \\
\hline 21 & 0.0026 & 0.0022 & 0.0016 & 0.9510 & & & & & & & \\
\hline
\end{tabular}

